\title{
Farmácia viva, o cuidado farmacêutico nas unidades básicas de saúde no Nordeste
}

\author{
Viva pharmacy, pharmaceutical care in basic health units in the Northeast
}

\author{
Roberta Silva de Almeida*, Jaissa Cristina de Bastos Nobre ${ }^{\bullet}$, Juliana Azevedo da Paixão ${ }^{\bullet}$ \\ Bacharelado em Farmácia, Universidade Salvador (UNIFACS), Salvador, Bahia, Brasil. *Autor para correspondência. E-mail: \\ betalmeida034@gmail.com
}

\begin{abstract}
Resumo: Introdução: O presente artigo relata a importância das farmácias vivas na atenção básica, pontuando sua trajetória e a sua contribuição para o Sistema Único de Saúde (SUS) através da inserção dos fitoterápicos pela portaria $\mathrm{N}^{\circ} 886$, desenvolvendo o cultivo, coleta e processamento de plantas medicinais regionais com segurança e eficácia nas práticas de manipulação e dispensação atendendo desta forma ao perfil epidemiológico da população. Revisão: Para realização do artigo, os métodos e os resultados utilizados para estudo foram estruturados na revisão bibliográfica narrativa que utiliza como fonte de pesquisa bases de dados eletrônicos, como Google Acadêmico, Scientific Electronic Lirary Online (Scielo), Revista de Saúde e Sites Governamentais como o Ministério da Saúde e Anvisa entre os anos de 2004 até 2021 na língua portuguesa. Discussão: Pontua-se a importância da assistência farmacêutica em conjunto com os multiprofissionais que constituem a base da Farmácia Viva, tornando relevante sua escrita para ampliar o conhecimento sobre o programa e as dificuldades enfrentadas para a continuidade do serviço. Considerações Finais: Conclui-se que o tema é de extrema importância para os usuários do Sistema Único de Saúde (SUS), pois, descreve a importância da Farmácia Viva e o cuidado farmacêutico na atenção primária, garantindo uma maior qualidade para o usuário durante seu acesso as plantas medicinais e aos fitoterápicos.
\end{abstract}

Palavras-chaves: plantas medicinais, farmácia viva, fitoterapia, etnofarmacologia, atenção básica e assistência farmacêutica.

\begin{abstract}
Introduction: This article reports the importance of live pharmacies in primary care, pointing out their trajectory and their contribution to the Unified Health System (SUS) through the insertion of herbal medicines through Ordinance No. 886, developing the cultivation, collection and processing of regional medicinal plants safely and effectively in handling and dispensing practices, thus meeting the epidemiological profile of the population. Review: To carry out the article, the methods and results used for the study were structured in the narrative bibliographic review that uses electronic databases as a research source, such as Academic Google, Scientific Electronic Lirary Online (Scielo), Revista de Saúde and Government Sites such as the Ministry of Health and Anvisa between 2004 and 2021 in Portuguese. Discussion: The importance of pharmaceutical care together with the multiprofessionals who form the basis of Pharmacy Viva is highlighted, making its writing relevant to increase knowledge about the program and the difficulties faced for the continuity of the service. Final Considerations: It is concluded that the topic is extremely important for users of the Unified Health System (SUS), as it describes the importance of Live Pharmacy and pharmaceutical care in primary care, ensuring greater quality for the user during their access to medicinal plants and herbal medicines.
\end{abstract}

Keywords: medicinal plants, live pharmacy, phytotherapy, ethnopharmacology, primary care and pharmaceutical assistance.

\section{Introdução}

A farmácia viva é compreendida pelo serviço de saúde da assistência farmacêutica, através do cultivo, processamento e manipulação magistral de plantas medicinais regionais. O projeto surgiu no Ceará em 1983 através da dedicação, estudo e pesquisas desenvolvidas pelo Professor Dr. Francisco José de Abreu Matos em conjunto com a Universidade Federal do Ceará (UFC), compreendendo que boa parte da população Nordestina passava por escassez nos serviços de saúde, utilizando plantas locais de uso popular como único recurso terapêutico (Prado et al.,2018; Randal et al., 2016). Segundo o Ministério da Saúde no Sistema Único de Saúde (SUS) através da portaria GM/MS n 886/2010, foi instituída a farmácia viva como um campo de atuação da 
assistência farmacêutica, sobre a gestão estadual, municipal ou do Distrito Federal, conforme orientações da Política Nacional de Práticas Integrativas e Complementares (PNPIC) e da Política Nacional de Plantas Medicinais e Fitoterápicos (PNPMF) (Brasil, 2013).

A Política Nacional de Práticas Integrativas e Complementares (PNPIC) recomenda em suas diretrizes a necessidade do provimento do acesso as plantas medicinais e fitoterápicos aos usuários do Sistema Único de Saúde (SUS) em especial nas unidades de saúde da família manipulando para isso um ou mais dos produtos a seguir: planta medicinal in natura, droga vegetal, fitoterápico e até o fitoterápico industrializado (Brasil ,2006; 2010).

Nesse aspecto, as farmácias vivas podem ser classificadas em três modelos conforme os serviços prestados à população. $\mathrm{O}$ modelo I atua com a planta medicinal in natura além da orientação sobre a forma correta de preparação até o seu uso caseiro em forma de Chás, o modelo II com a dispensação da droga vegetal e o modelo III com as preparações magistrais farmacotécnicas e fitoterápicas produzidas nas hortas oficiais (Bonfim et al., 2019; Brasil, 2012a).

Desta forma, temos a incorporação dos estudos etnobotânicos em coparticipação com pesquisas etnofarmacológicas; que preconizam uma linha de pesquisa relativamente nova que reúne informações a respeito da origem, indicação e ação terapêutica da droga de forma multidisciplinar sem prejulgamentos culturais, históricos ou acadêmicos, ampliando assim o olhar científico para pesquisas que auxiliaram os atuais e futuros tratamentos (Almeida, 2011).

Mesmo que a finalidade das plantas medicinais seja antiga na cultura popular, indígena, quilombola, agricultura familiar, populações ribeirinhas e até pelas figuras populares das parteiras, benzedeiras; No Sistema Único de Saúde (SUS) extraordinariamente na atenção básica sua inserção é recente se pararmos para comparar as ações inclusivas das farmácias vivas e a sua comedida divulgação mesmo tendo o potencial botânico e cultural inerente a divisão territorial do Brasil em especial no Nordeste (Alves et al., 2015; Antonio et al., 2013; Brasil, 2016).

Dentro desse contexto a contribuição da assistência farmacêutica reside, especialmente, em certificar condutas que objetivem uma maior promoção, proteção, e recuperação da saúde, do usuário como de sua comunidade; proporcionando o desenvolvimento e consequentemente a produção de medicamentos, atentando-se para a seu ciclo farmacêutico, garantindo assim qualidade dos insumos e serviços, acompanhamento e verificação de sua utilização, na compreensão para a aquisição de resultados efetivos para a ampliação do bem-estar da população (Brasil, 2004; 2008).

Conforme abordado, o tema proposto é de extrema importância para os usuários do Sistema Único de Saúde (SUS), pois, nos questiona sobre a importância da Farmácia Viva e o cuidado farmacêutico na atenção primária garantindo desta forma a seguridade com qualidade para o usuário durante seu acesso as plantas medicinais e aos fitoterápicos além dos cuidados oferecidos pela assistência farmacêutica pertinentes com a sua manipulação, prescrição e dispensação correlacionando com o uso racional dos mesmos, contribuindo desta forma para o enriquecimento cultural e terapêutico. Para tanto, tem como objetivo realizar através do estudo bibliográfico a compreensão da implantação da farmácia viva e sua importância no cuidado farmacêutico nas unidades básicas de saúde especialmente no Nordeste.

\section{Revisão}

O presente artigo trata de uma revisão bibliográfica narrativa que utiliza como fonte de pesquisa bases de dados eletrônicos, como Google Acadêmico, Scientific Electronic Lirary Online (Scielo), Revista de Saúde e Sites Governamentais como o Ministério da Saúde e Anvisa. Tendo como critério de rastreio os seguintes descritores: Plantas medicinais, Farmácia viva, Fitoterapia, Etnofarmacologia, Atenção básica e Assistência Farmacêutica, incluindo possíveis combinações entre as mesmas. Foram usados com critérios de inclusão a escolha de artigos científicos, Boletins, livros digitais, cadernos digitais e normas regulamentadoras; que descrevam sobre a importância da farmácia viva especialmente na atenção básica com a atuação da assistência farmacêutica, sobre o uso de medicamentos fitoterápicos na rede pública de saúde abordando a inclusão/ análise das farmácias vivas, como também artigos relacionados a farmacovigilância para as plantas medicinais e seus desafios.

\section{Farmácias vivas no sus}

Para entender a importância da inserção dessa "nova” prática de como pensar/tratar a doença e promover saúde temos que olhar para o passado visualizando, portanto, como sua história foi esculpida conforme Quadro 1 (Apresenta a linha do tempo referente a implantação das farmácias vivas no Brasil). 
Quadro 1. Linha do Tempo Farmácias Vivas no Brasil

\begin{tabular}{|c|c|c|}
\hline Ano & \begin{tabular}{|c|} 
Evento \\
\end{tabular} & Informações importantes \\
\hline 1978 & $\begin{array}{l}\text { Conferência Internacional Conferência Internacional } \\
\text { sobre Cuidados Primários de Saúde (Declaração de Alma- } \\
\text { Ata). }\end{array}$ & $\begin{array}{l}\text { Discursões e novas perspectivas sobre atenção primária em } \\
\text {-saúde. os princípios de Alma-Ata foram incorporados à } \\
\text { nova Constituição brasileira de } 1988 \text {, }\end{array}$ \\
\hline 1982 & $\begin{array}{l}\text { Central de Medicamentos do Ministério da Saúde } \\
\text { (PPPM/CEME) }\end{array}$ & $\begin{array}{l}\text { Criada para pesquisar os benefícios e toxidades das } \\
\text { preparações de uso popular com plantas medicinais. }\end{array}$ \\
\hline 1983 & Programa de Pesquisas de Plantas Medicinais (PPPM) & $\begin{array}{l}\text { Este programa substitui o (PPPM/CEME).O Programa de } \\
\text { Pesquisas de Plantas Medicinais (PPPM), conta com a } \\
\text { participação da ANVISA e da Fundação Oswaldo Cruz } \\
\text { (FIOCRUZ). }\end{array}$ \\
\hline 1986 & $8^{\text {a }}$ Conferência Nacional de Saúde & $\begin{array}{l}\text { Introdução de práticas alternativas de assistência à saúde } \\
\text { no âmbito dos serviços de saúde. }\end{array}$ \\
\hline 1988 & Comissão Interministerial de Planejamento (CIPLAN), & $\begin{array}{l}\text { Elaborou a Resolução } \mathrm{n}^{\circ} \text { 8/88 que regulamentou a } \\
\text { implantação da Fitoterapia nos Serviços de Saúde e nas } \\
\text { Universidades Federais nos procedimentos da assistência } \\
\text { médica. }\end{array}$ \\
\hline 1992 & $\begin{array}{l}\text { O Conselho Federal de Medicina reconhece a fitoterapia } \\
\text { como método terapêutico }\end{array}$ & Parecer n. ${ }^{\circ}$ 04/92 em 17 de janeiro de 1992 \\
\hline 1998 & $\begin{array}{l}\text { Subcomissão Nacional de Assessoramento em } \\
\text { Fitoterápicos (CONAFIT) }\end{array}$ & $\begin{array}{l}\text { Através da Portaria n. }{ }^{\circ} 665 \text { de } 1998 \text {, do Ministério produtos } \\
\text { fitoterápicos junto ao Sistema de Vigilância da Saúde é } \\
\text { criada a CONAFIT. Para orientar Secretaria de Vigilância } \\
\text { Sanitária, nos assuntos científicos e normativos envolvidos } \\
\text { na avaliação da eficácia e segurança do uso de produtos } \\
\text { fitoterápicos. }\end{array}$ \\
\hline 2003 & Relatório da $12^{\text {a }}$ Conferência Nacional de Saúde & $\begin{array}{l}\text { Discursões sobre a necessidade de investimento na } \\
\text { pesquisa e desenvolvimento de tecnologia para produção } \\
\text { de medicamentos a partir da flora brasileira. }\end{array}$ \\
\hline 2004 & Resolução n 338, Conselho Nacional de Saúde & $\begin{array}{l}\text { Aprovação da Política Nacional de Assistência } \\
\text { Farmacêutica que contempla a definição e pactuação de } \\
\text { ações intersetoriais que visam à utilização das plantas } \\
\text { medicinais e de medicamentos fitoterápicos no processo de } \\
\text { atenção à saúde, com respeito aos conhecimentos } \\
\text { tradicionais incorporados, embasamento científico, }\end{array}$ \\
\hline 2005 & $\begin{array}{l}1^{\circ} \text { Conferência Nacional de Medicamentos e Assistência } \\
\text { Farmacêutica. }\end{array}$ & $\begin{array}{l}\text { Realizado pelo Departamento de Assistência Farmacêutica } \\
\text { e Insumos Estratégicos (DAF/SCTIE), com aprovação de } 48 \\
\text { recomendações para uso fitoterápico. }\end{array}$ \\
\hline 2006 & $\begin{array}{l}\text { Política Nacional de Práticas Integrativas e } \\
\text { Complementares (PNPIC) no Sistema Único de Saúde } \\
\text { (SUS); } \\
\text { Política Nacional de Plantas Medicinais e Fitoterápicos } \\
\text { (PNPMF). }\end{array}$ & Portaria $\mathrm{N}^{\circ}$ 971/GM/MS para (PNPIC); \\
\hline 2008 & $\begin{array}{l}\text { Comitê Nacional de Plantas Medicinais e Fitoterápicos. } \\
\text { ANVISA coloca em vigor a Instrução Normativa-IN/05. }\end{array}$ & $\begin{array}{l}\text { Publicação da Portaria Interministerial } \mathrm{n}^{\circ} 2.960-\text { que } \\
\text { institui o Programa Nacional de Plantas Medicinais e } \\
\text { Fitoterápicos e cria o Comitê Nacional de Plantas } \\
\text { Medicinais e Fitoterápicos. } \\
\text { Divulgação de } 36 \text { plantas para fins medicinais. }\end{array}$ \\
\hline 2009 & $\begin{array}{l}\text { Diretoria de Assistência Farmacêutica publicou a } \\
\text { Relação de Plantas Medicinais de Interesse ao SUS } \\
\text { (RENISUS) }\end{array}$ & $\begin{array}{l}\text { Apresenta uma relação nacional de plantas com } 71 \\
\text { espécies vegetais de interesse para o SUS. }\end{array}$ \\
\hline 2010 & $\begin{array}{l}\text { Instituído no Sistema Único de Saúde (SUS), através dos } \\
\text { cuidados da gestão estadual, municipal ou do Distrito } \\
\text { Federal, a Farmácia Viva. }\end{array}$ & $\begin{array}{l}\text { Segundo Portaria } \mathrm{N}^{\circ} 886 \text {, de } 20 \text { de abril de } 2010 \text {, } \\
\text { sespeitando a legislação nacional do SUS e os padrões } \\
\text { técnicos para prestar essa assistência farmacêutica } \\
\text { conforme Resolução } N^{0} 338 \text {, do Conselho Nacional de } \\
\text { Saúde, de } 6 \text { de maio de } 2004 \text {. }\end{array}$ \\
\hline 2011 & Formulário Fitoterápicos da Farmacopeia Brasileira & $1^{\text {a }}$ edição, segundo a RDC nº 60 dos fitoterápicos \\
\hline 2012 & 12 Fitoterápicos registrados na RENAME & $\begin{array}{l}\text { Publicação da Referência Nacional para Medicamentos e } \\
\text { Insumos Complementares para a Assistência Farmacêutica } \\
\text { na Atenção Básica (Portaria nº 533/GM/MS). }\end{array}$ \\
\hline 2013 & Resolução -RDC - No 18, de 3 de Abril de 2013 & $\begin{array}{l}\text { Dispõe sobre as boas práticas de processamento e } \\
\text { armazenamento de plantas medicinais, preparação e } \\
\text { dispensação de produtos magistrais e oficinais de plantas } \\
\text { medicinais e fitoterápicos em farmácias vivas no âmbito do } \\
\text { Sistema Único de Saúde (SUS). }\end{array}$ \\
\hline
\end{tabular}




\begin{tabular}{|c|c|c|}
\hline $2012-2020$ & $\begin{array}{c}\text { Repasses financeiros para estruturação, consolidação e } \\
\text { fortalecimento de Arranjos Produtivos Locais. }\end{array}$ & $\begin{array}{c}\text { Inicia-se a seleção dos programas da FV no âmbito do } \\
\text { SUS, através de editais . }\end{array}$ \\
\hline 2021 & I Simpósio de Farmácias Vivas & $\begin{array}{c}\text { Composição da Associação Brasileira Farmácia Viva com } \\
31 \text { membros fundadores para dar suporte e unificar as } \\
\text { ações dos projetos ativos. }\end{array}$ \\
\hline
\end{tabular}

Fonte: Autores.

Através da Conferência Internacional em Alma-Ata iniciam-se discursões e novas perspectivas sobre atenção primária em saúde, promovida pela Organização Mundial de Saúde (OMS) e pela UNICEF, alertando para a urgência dos governos em auxiliar e motivar a saúde dos povos do mundo através de formulação de políticas e normas. No final da década de 70, a OMS, desenvolve o Programa de Medicina Tradicional recomendando aos Estados-membros a elaboração de políticas públicas objetivando a incorporação da medicina tradicional e da medicina complementar alternativa no sistema nacional de atenção à saúde (Brasil, 2013; Gonçalves et al., 2020).

Durante a Assembleia Mundial de Saúde, as recomendações feitas em Alma-Ata são reiteradas com o objetivo de apoiar a criação de programas que promovessem ações voltadas para a identificação, avaliação, preparo, cultivo e conservação das plantas utilizadas na medicina tradicional garantindo desta forma uma maior qualidade para as drogas provenientes dos medicamentos tradicionais, as quais são extraídas de plantas através do uso das boas práticas de fabricação (Brasil, 2006b).

Um ano após a Assembleia Mundial de Saúde, a OMS convoca a primeira conferência internacional sobre Plantas Medicinais em Chiang Mai, na Tailândia. A qual, promoveu a adoção da Declaração de Chiang Mai que insere o uso racional de plantas medicinais de maneira definitiva no sistema de saúde pública. A fitoterapia passa a ter destaque e valorização frente ao uso dos medicamentos sintéticos industrializados cujo custo monetário é superior aos fitoterápicos muitas vezes inviabilizando a adesão ao tratamento para a população economicamente desamparada especialmente em países em desenvolvimento (Brasil, 2006b).

Após as manifestações internacionais no Brasil iniciam-se pesquisas e programas que vislumbram a inserção dos fitoterápicos para o tratamento da população. Iniciamos os estudos sobre os benefícios e toxidades das preparações de uso popular com plantas medicinais com a Central de Medicamentos do Ministério da Saúde (PPPM/CEME) que foi substituída pelo Programa de Pesquisas de Plantas Medicinais (PPPM), com a participação da ANVISA e da Fundação Oswaldo Cruz (FIOCRUZ).Com a implantação da PPPM, sobre uso popular das plantas medicinais existentes no Brasil, almeja-se obter os possíveis princípios ativos que servissem para o preparo de medicamentos fitoterápicos, após três anos da implementação do programa, ocorre em Brasília, a $8^{\text {a }}$ Conferência Nacional de Saúde "introdução de práticas alternativas de assistência à saúde no âmbito dos serviços de saúde, possibilitando ao usuário o acesso democrático de escolher a terapêutica preferida"(CONFERÊNCIA NACIONAL DE SAÚDE, 1986). Com o desenvolvimento e aprimoramento das pesquisas e dos debates científicos a Comissão Interministerial de Planejamento (CIPLAN), elaborou a Resolução no $8 / 88$ que regulamentou a implantação da Fitoterapia nos Serviços de Saúde e nas Universidades Federais nos procedimentos da assistência médica (Brasil,2006b; Castro \& Figueiredo, 2019; Gonçalves et al., 2020).

A fitoterapia passa a ser reconhecida pelo conselho Federal de Medicina como método terapêutico através do Parecer $n .^{\circ}$ 04/92, sendo supervisionada pelo Estado através da Vigilância Sanitária. Instituindo e normatizando o registro de Sanitário em 1995 com a Portaria n. ${ }^{\circ}$ 06/SVS. Através da Portaria n. ${ }^{\circ} 665$ de 1998, do Ministério produtos fitoterápicos junto ao Sistema de Vigilância da Saúde, é criada a Subcomissão Nacional de Assessoramento em Fitoterápicos (CONAFIT) que tinha a incumbência de orientar a Secretaria de Vigilância Sanitária, nos assuntos científicos e normativos envolvidos na avaliação da eficácia e segurança do uso de produtos fitoterápicos, além de promover estudos certificados sobre a farmacovigilância fomentando as pesquisas clínicas (Brasil, 2006b; Gonçalves et al., 2020).

Durante o $1^{\circ}$ Conferência Nacional de Medicamentos e Assistência Farmacêutica, realizado pelo Departamento de Assistência Farmacêutica e Insumos Estratégicos (DAF/SCTIE),em Brasília foram apresentados 68 recomendações das quais só 48 foram aprovadas, dentre elas a criação de unidades nos estados para elaboração de medicamentos fitoterápicos, preferindo as espécies vegetais nativas desenvolvendo a Política Nacional de Plantas Medicinais e Fitoterápicos, com financiamento, assegurando desta maneira o ingresso aos produtos certificados; regulamentando desta forma o uso das plantas medicinais e dos medicamentos fitoterápicos na Assistência Farmacêutica, como elemento da Atenção Básica (Brasil, 2006b).

Com a portaria $N^{\circ}$ 971/GM/MS,2006, foi instituída a Política Nacional de Práticas Integrativas e Complementares (PNPIC) no Sistema Único de Saúde (SUS), no mesmo ano através do Decreto $\mathrm{N}^{\circ} 5.813$, aprova-se a Política Nacional de Plantas Medicinais e Fitoterápicos (PNPMF), a qual incentivou de forma 
segura o uso das plantas medicinais, sendo criado no ano de 2008 o Comitê Nacional de Plantas Medicinais e Fitoterápicos; No mesmo ano a ANVISA coloca em vigor a Instrução Normativa-IN/05, com uma lista de 36 plantas para fins medicinais.om os avanços nos estudos a Diretoria de Assistência Farmacêutica publica em 2009 a Relação de Plantas Medicinais de Interesse ao SUS (RENISUS) apresentando uma relação nacional com 71 espécies vegetais, as quais devem ser estudadas para garantir segurança e eficácia em seu uso ( Brasil, 2012a ;Gonçalves et al., 2020).

Observando a necessidade de ampliação das demandas sociais e locais, em relação à oferta de fitoterápicos e plantas medicinais foi instituído no Sistema Único de Saúde (SUS), através dos cuidados da gestão estadual, municipal ou do Distrito Federal, a Farmácia Viva, segundo Portaria № 886, de 20 de abril de 2010, respeitando a legislação nacional do SUS e os padrões técnicos para prestar essa assistência farmacêutica conforme Resolução No 338, do Conselho Nacional de Saúde, de 6 de maio de 2004 (Almeida,2011; Brasil, 2010).

A Agência Nacional de Vigilância Sanitária através da RDC $N^{\circ} 18$, concebe por farmácias vivas (FV) aquelas instituídas pela "Portaria $\mathrm{n}^{\circ}$ 886, de 20 de abril de 2010, do Ministério da Saúde, que realizam as etapas de cultivo, processamento, armazenamento de plantas medicinais, preparação e dispensação de produtos magistrais e oficinais de plantas medicinais e fitoterápicos” (Brasil, 2013). Essa portaria delibera os requisitos minimamente necessários para as práticas das atividades de manipulação de plantas medicinais e fitoterápicos, garantindo qualidade, segurança e a efetividade alinhando esta prática com o uso seguro e racional. Sendo de fundamental importância os cuidados/controles das instalações, equipamentos e dos profissionais como também o gerenciamento da qualidade para a matéria-prima e o seu armazenamento através da avaliação farmacêutica desde a prescrição até a dispensação das preparações, além dos cuidados necessários durante a atenção farmacêutica aos usuários no âmbito das farmácias vivas que tenham sua própria horta oficial ou comunitária e que sejam dispensados pelo SUS (Brasil, 2013).

A farmácia viva é composta por três modelos conforme seu nível de complexidade e proposta do projeto para implementação, onde o nível I realiza o cultivo e o acesso as plantas medicinais in natura em hortas comunitárias ou unidades do SUS com orientação sobre como preparar e usar corretamente os tratamentos caseiros, o nível II refere-se a produção e dispensação das plantas medicinais secas destinadas para uso nas Unidades Básicas de Saúde e por último o nível III que corresponde a preparação dos medicamentos fitoterápicos para dispensação nas unidades do SUS. Podemos ressaltar, que o programa Farmácia Viva pode atuar separadamente como também de forma sincrônica unicamente no nível III, o qual pode abranger os dois primeiros como pode-se verificar nas Unidades do Ceará graças a dedicação do Professor Matos e de sua equipe (Bonfim et al., 2019; Rufino et al., 2018).

Segundo dados divulgados em endereço eletrônico do Ministério da Saúde já foram apoiados 138 projetos envolvendo assistência farmacêutica com plantas medicinais e fitoterápicos entre os anos de 2012 até 2019. No ano de 2020 por meio de edital, SCTIE/MS $n^{\circ} 2 / 2020$, realizou processo seletivo dos projetos para estruturação de Farmácias Vivas na atenção primária, sendo apoiadas 10 secretarias municipais de saúde, durante 36meses, a saber: Araraquara (SP), Salvador (BA), Varginha (MG), Afogados da Ingazeira (PE), Cachoeiras de Macacu (RJ), Brumadinho (MG), Pindamonhangaba (SP), Quijingue (BA), São Cristóvão (SE) e Caruaru (PE) (André, 2021).

\section{Atuação farmacêutica na farmácia viva}

Segundo a resolução $n^{\circ} 18 / 2013$ (Brasil, 2013) a assistência farmacêutica é o um conjunto de ações e ocupações relacionados aos medicamentos, apoiando as demandas trazidas pela comunidade em torno da saúde. Cabendo as suas funções o abastecimento dos medicamentos desde a aquisição, conservação perpassando pelo controle de qualidade. $O$ acompanhamento e a constante avaliação da utilização ajudam na propagação de informações seguras para a equipe de saúde e comunidade.

A assistência social farmacêutica na farmácia viva, na atenção primária, incentiva a promoção do registro das plantas medicinais através de entrevistas de abordagem Fitoterápica (Questionário elaborado pela Coordenação do Projeto conforme a realidade de cada Município) entre a comunidade para selecionar plantas medicinais regionais a fim de promover a produção das mesmas com o cultivo orgânico das mudas certificadas em horta oficiais ou comunitárias segundo critérios técnicos, considerando os aspectos agronômicos e farmacêuticos até a sua manipulação e dispensação na rede pública com o apoio / financiamento do Governo Estadual e Federal para isso os medicamentos fitoterápicos devem estar listados para sua inclusão na Relação de Plantas Medicinais de Interesse ao SUS (RENISUS) (Gouveia \& Simionato, 2019).

Subsidiar atenção e escuta ativa à comunidade como um todo faz parte das atribuições da equipe de saúde, a qual para exercê-la com propriedade deve planejar suas ações baseada na cultura da população imbuindo- 
se dos recursos existentes para execução de propostas que desenvolvam a melhoria da saúde. Desta forma, os conhecimentos a respeito das plantas medicinais e suas propriedades, bem como a sua elaboração, dose e indicação devem fazer parte deste procedimento, pois, são tratamentos utilizados pela comunidade em seu processo de busca pela saúde (Guimarães et al., 2017; Soares et al., 2018).

Uma das bases da farmácia viva, é composta pela equipe multiprofissional (médicos, enfermeiras, farmacêuticos, agrônomos, jardineiros, biólogos, nutricionista, agentes comunitários etc.); contudo, três profissionais são extremamente importantes para extensão do projeto e sua fundamentação: o agrônomo e o jardineiro que se responsabilizam pelo plantio das espécies, o médico que tem propriedade para diagnosticar e prescrever composições e o farmacêutico que presta serviços de orientação, manipulação magistral além do controle de qualidade dos fitoterápicos prescritos desde a coleta até o preparo (Brasil, 2016b; Prado et al., 2018).

Para tanto o levantamento e a escolha das plantas a serem usadas podem ser realizadas através de consultas, visitas domiciliares, grupos de palestras entre os profissionais e a comunidade usuária da unidade básica de saúde. Esse resgate do conhecimento popular será usado posteriormente para uma ação educativa com compartilhamento de explicações cientificas sobre o uso correto e adequado das plantas medicinais (Gouveia \& Simionato, 2019).

Como nas preparações industrializadas, as plantas medicinais da farmácia viva passam pelo planejamento dos vegetais nomeados tendo em vista a sazonalidade dos casos clínicos identificados na comunidade que busca o atendimento na Atenção Básica de Saúde como os distúrbios estomacais, dor de cabeça, resfriados, edemas, ansiedade, expectorantes, cicatrizantes etc., além de outras variantes como surtos, demandas de outras unidades e até o fornecimento de mudas ou insumos para a sua manipulação em laboratório (Castro \& Figueiredo, 2019).

Nove espécies foram aprovadas para uso pela ANVISA e até pela PPPM (Programa de Pesquisa Planta Medicinais) para a fabricação de medicamentos fitoterápicos, conforme comprovação de sua eficiência no tratamento das doenças entre elas: Alcachofra (Cynara scolymus), Aroeira (Schinus terebentthifolius), Cáscara sagrada (Rhamnus purshiana), Garra do diabo (Harpagophytum procumbens), Guaco (Mikania laevigata), Unha de gato (Uncaria tomentosa) e o composto isoflavona de soja (Castro\& Figueiredo, 2019).

Conforme a RDC $n^{\circ} 18 / 2013$ a Farmácia Viva deve ser aprovada pela inspeção sanitária para que possa desenvolver os produtos Magistrais, para tanto deve estar regularizada com a Vigilância Sanitária local, possuir autorização de funcionamento de empresa expedida pela Anvisa além de atender as disposições da resolução; Portanto o farmacêutico possui orientações normatizadas sobre as boas práticas de processamento, higienização, controle de qualidade, armazenamento e rotulagem de plantas medicinais, preparação e dispensação de produtos magistrais e oficinais de plantas medicinais e fitoterápicos provenientes de hortas, hortos oficiais ou comunitários e até de fornecedores qualificados (agricultores cadastrados no programa) para fornecer os insumos vegetais para o programa farmácia Viva no âmbito do Sistema Único de Saúde (SUS) sendo proibido a comercialização de plantas medicinais e fitoterápicos. Segundo orientações especificadas nesta resolução as plantas medicinais utilizadas na Farmácia Viva devem ser identificadas botanicamente por profissional habilitado ou através de aquisição de mudas fornecidas por hortos oficiais que seguem controles de qualidade. Contudo, para os demais fornecedores de plantas medicinais é obrigatório a apresentação do certificado de análise, o qual deve conter informações precisas e todas as especificações do responsável técnico e do fornecedor /fabricante.

Portanto, a atuação farmacêutica deve desenvolver ações desde a escolha, produção, prescrição e dispensação até a informação e conscientização do público externo/pacientes sobre a eficácia e o uso racional dos fitoterápicos e das plantas medicinais sem que para isso tenha que suspender temporariamente ou em definitivo o tratamento tradicional industrial, mas, ajustando-os e/ou harmonizando-os para um melhor retorno da resposta do paciente. Podendo desenvolver grupos entre as unidades da atenção primária para o compartilhamento dos resultados; fomentando a instigação para a pesquisa propiciando desta forma o registro das plantas territoriais em conjunto com a sua farmacovigilância (Antônio et al., 2013).

\section{Discussão}

Com a instauração da Política Nacional de Práticas Integrativas e Complementares juntamente com a Política Nacional de Plantas Medicinais, as quais em conjunto com as manifestações universitárias que através de ações voltadas para a extensão e pesquisa no Nordeste especialmente no Ceará, apoiados pelas determinações da Organização Mundial da Saúde (OMS) percebe-se avanços, mesmo que tímidos, na inserção do programa, através das legislações sanitárias, decretos e normas regulamentadoras que promoveram o 
desenvolvimento da pesquisa com a valorização da fitoterapia e das plantas medicinais através da farmácia viva na atenção primária (Antonio, et al., 2014).

Os trabalhos desenvolvidos pelas Farmácias Vivas garantem à população dois tipos de assistência abrangendo a área de fitoterapia: A preparação de fitoterápicos segundo técnicas farmacêuticas de boas práticas e a dispensação na rede pública de saúde com orientação sobre o uso correto de plantas medicinais com o apoio farmacêutico, através de hortos com espécies vegetais regionais que possuam certificação botânica, sendo garantido, dessa forma, a eficácia, a segurança e a qualidade. Portanto, é de competência desde o cultivo até sua dispensação conforme as normas regulamentadoras sanitárias e ambientais dos órgãos regulamentadores como a ANVISA especialmente para a manipulação magistral (Brasil, 2013). Para tanto, alguns desafios devem ser ultrapassados pelos profissionais de saúde como: conhecer as indicações e formas de uso das plantas e dos fitoterápicos, as reações alérgicas ou tóxicas, as reações derivadas de interações medicamentosas ou de superdosagem, controle de qualidade através das boas práticas de produção e manipulação, os cuidados com o armazenamento e até com a rotulação dos medicamentos manipulados (Niehues et al., 2011).

Segundo dados do Ministério da Saúde temos 10 Secretarias aprovadas através de Edital/2020 para incentivo e produção de fitoterápicos através do projeto Farmácia viva, sendo que deste total somente 02 são para a Bahia (Salvador; Quijingue), dois para Pernambuco (Afogados da Ingazeira; Caruaru) e um para o estado de Sergipe (São Cristóvão) uma contagem abaixo das expectativas quando vislumbramos o potencial das plantas nordestinas apresentadas pelas pesquisas iniciadas no Ceará. Qualquer Secretaria de Saúde pode participar da seleção do edital, contudo devem comprovar sua capacidade em executar o projeto desde o cultivo até dispensação dentro do prazo estipulado da vigência do programa.

Em Salvador podemos destacar o projeto Farmácia Viva do curso de Farmácia da Universidade Federal da Bahia (UFBA), o qual foi aprovado em 14 de outubro de 2020 em parceria com Secretaria Municipal de Saúde de Salvador. O Projeto será estruturado com dois hortos farmacobotânicos localizados no centro de atenção Psicossocial (CAPS) III e no Horto Farmácia da Terra da Faculdade de Farmácia da UFBA. Onde as etapas de produção serão realizadas nas instalações da Faculdade, sendo distribuídos na Farmácia Universitária e nas demais farmácias da rede municipal em meados do ano de 2022 (Ribas, 2020). No território Cearense através da Universidade Federal do Ceará (UFC) temos a matriz da farmácia viva, Horto de plantas medicinais da UFC, idealizado e criado pelo professor já falecido Francisco José de Abreu Matos que mantem suas atividades com desde a produção, pesquisa e dispensação dos fitoterápicos regionais, o Governo do Estrado do Ceará também matem o Núcleo de Fitoterápicos (Nufito) e em Fortaleza temos a parceria entre a prefeitura Municipal de Fortaleza com a Universidade de Fortaleza (Unifor) com a Farmácia Viva Lucia Gurgel referência na produção dos fitoterápicos.

Vale ressaltar que no território Brasileiro, cinco regiões são consideradas ricas em espécies medicinais: Floresta Amazônica, Mata Atlântica, Pantanal Mato-grossense, Cerrado e Caatinga. Dentro destas regiões, algumas possuem plantas medicinais que não possuem pesquisas sobre sua composição química, farmacológica, ou toxicológica as quais, são popularmente indicadas para tratamentos (Almeida, 2011). O Bioma da Caatinga "Mata Branca" por exemplo carece de investimentos com pesquisa e preservação das espécies que sofrem com o desmatamento, as queimadas e o crescimento desorganizado das populações mais carentes. (Magalhães, 2020).

As farmácias vivas convivem com muitas dificuldades e com a ameaça constante de extinção dos projetos em virtude da falta de um plano de ação do Governo Federal em forma de lei que possa garantir verbas efetivas para manutenção das atividades e dos padrões técnicos-científicos cujo objetivo é minimizar subnotificações clínicas e desenvolver maior ênfase na pesquisa e consequentemente seu registro e divulgação (Brasil, 2012), essas questões compuseram um dos inúmeros tópicos dos debates trazidos durante o I simpósio de Farmácias vivas ocorrido no ano de 2021 que resultou na composição da Associação Brasileira Farmácia Viva com 31 membros fundadores para dar suporte e rumo aos novos passos da longa estrada que se estende para o projeto farmácia viva. Contudo, podemos visualizar que este inconstante apoio financeiro pode proporcionar contratempos significativos na aplicação e desenvolvimento do programa farmácia viva na atenção primária, especialmente no Nordeste, os quais incluem pouco investimento em estudos e inovação de plantas medicinais, atraso nos projetos de produção dos fitoterápicos e das plantas medicinais e enfraquecimento da política nacional das plantas medicinais. Desencadeando um déficit no registro de toxidade do uso das plantas medicinais e suas interações farmacológicas, falta de qualificação de recursos humanos (médicos, enfermeiros) além do desperdício da verba pública quando interrompe -se um programa que demanda de tempo e equipamentos para execução (Antonio et al., 2014). 
Portanto, a adesão dos fitoterápicos e das plantas medicinais nas unidades básicas de saúde no Nordeste vem para ampliar os cuidados com a prevenção e o alívio das doenças como também para empoderar o conhecimento popular que perpassam pelos valores, crenças e culturas de forma cativante, pois, compartilham lembranças, conhecimentos e porque não histórias. Diferentemente do que se observa nos tratamentos alopáticos (Soares et al., 2018). Levando-se sempre em conta a toxidade derivada de medicamentos elaborados pelas plantas medicinais, os quais não podem ser considerados de forma simplória pois, representam um preocupante problema para a saúde pública. Princípios ativos das plantas medicinais podem desenvolver reações adversas quando interagem com alimentos, outros medicamentos e até pelas características do paciente como idade, sexo, condições fisiológicas (Balbino \& Dias, 2010).

Em consonância aos dados expostos, extraídos das pesquisas feitas nos artigos, os quais ainda são poucos frente a importância científica, fica claro que a prática farmacêutica está contida na dinâmica da assistência farmacêutica da farmácia viva, pois, trata-se do compromisso assumido com corresponsabilidade para a promoção e recuperação da saúde de forma interativa entre a equipe de saúde e o paciente objetivando uma farmacoterapia que desenvolva uma melhor qualidade de vida para a prevenção e até a recuperação da saúde dentro das perspectivas biopsicossociais (Brasil, 2013).

\section{Considerações finais}

De acordo com o presente estudo realizamos um levantamento bibliográfico sobre a importância e aplicação da farmácia viva amparados pelo cuidado farmacêutico nas unidades básicas de saúde especialmente no Nordeste, descrevendo sua trajetória de inclusão no Sistema Único de Saúde (SUS) e como esta pode ajudar na adesão ao tratamento da população, em especial aos pacientes de baixa renda. Propiciando a compreensão da atuação farmacêutica na atenção primária e na estruturação da farmácia viva apresentando suas dificuldades de execução e manutenção dos projetos.

\section{Agradecimentos}

Agradecemos em memória aos esforços do prof. Francisco José de Abreu Matos e de todos os profissionais que lutam e se dedicam pela melhoria da saúde, especialmente nas comunidades mais carentes do nosso amado nordeste.

\section{Referência}

André, C. 2021. Ministério da Saúde apoia ampliação de Farmácias Vivas em todo o paíshttps://www.gov.br/saude/pt-br/assuntos/noticias/ministerio-da-saude-apoia-ampliacao-de-farmaciasvivas-em-todo-o-pais 18/02/2021.

Almeida, M. Z. 2011. Plantas Medicinais (3rd ed.). Salvador, BA: EDUFBA.

Alves, P. J. J., Lima, C. C., Santos, D. B., \& Bezerra, P. D. F. 2015. Conhecimento popular sobre plantas medicinais e o cuidado da saúde primária: um estudo de caso da comunidade rural de Mendes, São José de Mipibu/RN. Carpe Diem: Revista Cultural E Científica Do UNIFACEX, 13(1), 136-156.

Antonio, G. D., Tesser, C. D., \& Moretti, P. R. O. 2013. Contribuições das plantas medicinais para o cuidado e a promoção da saúde na atenção primária. Interface - Comunicação, Saúde, Educação, 17(46), 615-633.

Antonio, G. D.; Tesser, C. D., \& Moretti,P. R. O. 2014.Fitoterapia na atenção primária à saúde. Rev Saúde Pública.

Balbino, E., \& Dias, M. F. 2010. Farmacovigilância: um passo em direção ao uso racional de plantas medicinais e fitoterápicos. Revista Brasileira de Farmacognosia, 20(6), 992-1000.

Brasil, Agência Nacional de Vigilância Sanitária [ANVISA]. 2013. Resolução RDC no 18, de 3 de abril de 2013. Dispõe sobre as boas práticas de processamento e armazenamento de plantas medicinais, preparação e dispensação de produtos magistrais e oficinais de plantas medicinais e fitoterápicos em farmácias vivas no âmbito do Sistema Único de Saúde (SUS). Diário Oficial da União, Brasília, DF. Seção 1.http://bvsms.saude.gov.br/bvs/saudelegis/anvisa/2013/rdc0013_14_03_2013.pdf

Brasil, Ministério da Saúde. 2004. Conselho Nacional de Saúde. Resolução no 338, de 6 de maio de 2004. Aprova a Política Nacional de Assistência Farmacêutica. Diário Oficial da União, Brasília, DF, n. 96, p.52-53, Seção 1. https://bvsms.saude.gov.br/bvs/saudelegis/cns/2004/res0338_06_05_2004.html

Brasil, Ministério da Saúde. 2012. Secretaria de Atenção à Saúde. Departamento de Atenção Básica. Práticas integrativas e complementares: plantas medicinais e fitoterapia na Atenção Básica/Ministério da Saúde. 
Secretaria de Atenção à Saúde. Departamento de Atenção Básica. Brasília, DF: Ministério da Saúde.156 p : il. - (Série A. Normas e Manuais Técnicos) (Cadernos de Atenção Básica; n. 31) https://bvsms.saude.gov.br/bvs/publicacoes/praticas_integrativas_complementares_plantas_medicinais_c ab31.pdf

Brasil, Ministério da Saúde. 2008. Secretaria de Atenção à Saúde. Departamento de Atenção Básica. Práticas Integrativas e Complementares em saúde: uma realidade no SUS. Revista Brasileira Saúde da Família, 9, 7076. http://189.28.128.100/dab/docs/publicacoes/revistas/revista_saude_familia18_especial.pdf

Brasil, Ministério da Saúde. 2006. Secretaria de Ciência, Tecnologia e Insumos Estratégicos. Departamento de Assistência Farmacêutica. Política nacional de plantas medicinais e fitoterápicos / Ministério da Saúde, Secretaria de Ciência, Tecnologia e Insumos Estratégicos, Departamento de Assistência Farmacêutica. Brasília: Ministério da Saúde, 2006. 60 p. - (Série B. Textos Básicos de Saúde) https://bvsms.saude.gov.br/bvs/publicacoes/politica_nacional_fitoterapicos.pdf

Brasil, Ministério da Saúde. 2006. Secretaria de Atenção à Saúde. Departamento da Atenção Básica. Política Nacional de Práticas Integrativas e Complementares no SUS - PNPIC. Brasília, DF: Ministério da Saúde; 2006. https://bvsms.saude.gov.br/bvs/publicacoes/pnpic.pdf

Brasil, Ministério da Saúde. 2016. Secretaria de Ciência, Tecnologia e Insumos Estratégicos. Departamento de Assistência Farmacêutica. Política e Programa Nacional de Plantas Medicinais e Fitoterápicos / Ministério da Saúde, Secretaria de Ciência, Tecnologia e Insumos Estratégicos, Departamento de Assistência Farmacêutica. Brasília, DF: $\quad$ Ministério da $\quad$ Saúde, 2016.190 p. https://bvsms.saude.gov.br/bvs/publicacoes/politica_programa_nacional_plantas_medicinais_fitoterapicos.pdf

Brasil, Ministério da Saúde. 2010. Gabinete do Ministro. Portaria no 886, de 20 abril de 2010. Institui a Farmácia Viva no âmbito do Sistema Único de Saúde (SUS). Diário Oficial da União, Brasília, DF, n. 75, p.75,. Seção 1. http://bvsms.saude.gov.br/bvs/saudelegis/gm/2010/prt0886_20_04_2010.html

Bonfim, D. Y. G., Bandeira, M. A. M., Gomes, A. B., Brasil, A. R. L., Magalhães, K. do N., \& Sá, K. M. 2019. Diagnóstico situacional das farmácias vivas existentes no Estado do Ceará. Journal of Management \& Primary Health Care, 2179-6750.

Castro, M. R., \& Figueiredo, F. F. 2019. Saberes Tradicionais, Biodiversidade, Práticas Integrativas E Complementares: O Uso De Plantas Medicinais No Sus. Hygeia - Revista Brasileira de Geografia Médica e da Saúde, 15(31).

Guimarãe, J., \& Medeireiro, J.C., Vieira, L. A. 2017. Farmácia viva sus/betim - um relato de experiencia exitosa na implantação da fitoterapia no SUS. In Anais CONGREPICS. Campina Grande: Realize Editora

Gonçalves, R. N., Gonçalves, J. R. D. S. N., Buffon, M. D. C. M., Negrelle, R. R. B., \& de Azevedo Mazza, V. 2020. Os marcos legais das políticas públicas de plantas medicinais e fitoterápicos no Brasil. Revista de APS, 23(3).

Gouveia, G. D. A., \& Simionato, C. 2019. Plantas medicinais e fitoterapia na tenção básica. Universidade Federal de Santa Catarina, Núcleo Telessaúde Santa Catarina; Dados eletrônicos Flofrianópolis;CCS/UFSC,42p.https://ares.unasus.gov.br/acervo/html/ARES/14863/1/Apostila_Fitoterapia _N\%C3\%BAcleo\%20Telessaude\%20SC\%20UFSC.pdf

Magalhães, K. N.; Bandeira, M. A. M., \& Monteiros, M. P. 2020. Plantas Medicinais da Caatinga do Nordeste Brasileiro: etnofarmacopeia do Professor Francisco José de Abreu Matos. - Fortaleza: Imprensa Universitária. http://www.repositorio.ufc.br/handle/riufc/42962

Niehues, J., Bonetti, P., Souza, M. R. D., Maia, A. L., Piovezan, A. P., \& Peters, R. R. 2011. Levantamento etnofarmacológico e identificação botânica de plantas medicinais em comunidades assistidas por um serviço de saúde. Arq. Catarin. Med, 40, 34-39.

Prado, M. A. S. dos A., Matsuok, J. T., \& Giotto, A. C. 2018. Importância das Farmácias Vivas no âmbito da produção dos medicamentos fitoterápicos. Revista De Iniciação Científica E Extensão, 1(1), 32-37.

Randal, V. B., Behrens, M. D. D., \& Pereira, A. M. S. 2016. Farmácia Da Natureza: Um Modelo Eficiente De Farmácia Viva. Revista Fitos, 10(1), 73-76.

Ribas, C. 2020. Farmácia Viva: UFBA Abrigará Unidade de Pesquisa e Produção de Medicamentos fitoterápicos - Edgar digital.

Rufino, L. L., Rojas, G. G., Bandeira, M. A. M., Souza, J. R. F., \& Reis, J.N. P. 2018. Prática Das Farmácias Vivas Do Município De Fortaleza, Ceará, E A Necessidade De Uma Ação De Extensão Sistêmica. Extensão Rural, DEAER - CCR - UFSM, Santa Maria, v.25(4). 
Soares, A. Á. P., Rodrigues, A. C., de Araújo Neto, J. H., Cavalcante, A. L. C., Melo, O. F., \& Siqueira, R. M. P. (2018). Aceitação de fitoterápicos por prescritores da atenção primária à saúde. SANARE-Revista de Políticas Públicas, 17(2), 40-48.

\section{Minicurrículo}

Roberta Silva de Almeida. Estudante do Curso de Farmácia $8^{\circ}$ semestre da UNIFACS.

Jaissa Cristina de Bastos Nobre. Estudante do Curso de Farmácia $8^{\circ}$ semestre da UNIFACS.

Juliana Azevedo da Paixão. Graduação em Farmácia pela Universidade Federal da Bahia. Especialista em Farmacologia Aplicada a Prática Clínica pela AVM-Faculdade Integrada, mestre em Recursos Genéticos Vegetais pela Universidade Estadual de Feira de Santana. Atualmente faz doutorado em Química pela Universidade Federal da Bahia e é docente do curso de Farmácia da Universidade Salvador-UNIFACS.

Como citar: Almeida, R.S., Nobre, J.C.B., \& Paixão, J.A. 2021. Farmácia viva, o cuidado farmacêutico nas unidades básicas de saúde no Nordeste. Pubsaúde, 7, a286. DOI: https://dx.doi.org/10.31533/pubsaude7.a286

Recebido: 12 nov. 2021.

Revisado e aceito: 19 nov. 2021.

Conflito de interesse: os autores declaram, em relação aos produtos e companhias descritos nesse artigo, não ter interesses associativos, comerciais, de propriedade ou financeiros que representem conflito de interesse.

Licenciamento: Este artigo é publicado na modalidade Acesso Aberto sob a licença Creative Commons Atribuição 4.0 (CC-BY 4.0). 